References:

[1] Ploegh, H. L. (1998). Science, 280, 248-53.

[2] Benedict, C. A., Butrovich, K. D., Lurain, N. S., Corbei,1 J., Rooney, I., Schneider, P., Tschopp, J., Ware, C. F. (1999). Journal of Immunology, 162, 6967-6970.

Keywords: immunomodulation, HCMV UL144, glycosylation
MS04-P13

\section{Structure-based cofactor binding properties of dihydrodipicolinate reductase (PaDHDPR) from Panisporosarcina sp. TG-14}

Chang Woo Lee ${ }^{1}$, Jun Hyuck Lee ${ }^{2}$, Sun-Ha Park ${ }^{3}$

1. Polar Science, University of Science and Technology, Incheon, Korea South

2. Unit of Polar Genomics, Korea Polar Research Institute, Daejeon, Korea South

3. Unit of Polar Genomics, Korea Polar Research Institute, Incheon, Korea South

email: justay@kopri.re.kr

Dihydrodipicolinate reductase (DHDPR) is a key enzyme for the Diaminopimelate (DAP) and Lysine synthesis pathway by reducing the dihydrodipicolinate (DHDP) into tetrahydrodipicolinate (THDP) using $\mathrm{NAD}(\mathrm{P}) \mathrm{H}$ as a cofactor. It is known that DHDPR uses both NADPH and/or NADH as cofactor but the structural basis of its cofactor specificity is still unclear. In this study, we found that PaDHDPR from Paenisporosarcina sp. TG-14 has a strong preference for NADPH compared with NADH based on isothermal titration calorimetry and enzyme activity assay. To better understand the enzymatic mechanism of PaDHDPR in molecular level, the three crystal structures of unliganded, DPA (dipicolinate; a competitive inhibitor of DHDPR) bound and ternary (NADPH and DPA) complexed PaDHDPR were determined at $1.8,1.8$ and $2.1 \AA$ resolutions, respectively. Comparison of these PaDHDPR structures with previously reported other DHDPR structures reveals that only ternary complex PaDHDPR structure shows completely closed conformation suggesting both substrate and nucleotide co-factor are essential for the domain movement. Moreover, NADPH binding induced local conformation changes in the $\mathrm{N}$-terminal long loop region (residues 34-59) in PaDHDPR and the His35 and Lys36 residues located in this loop region make strong interaction network with the 2 '-phosphate group of NADPH. These specific interactions may allow PaDHDPR to have a strong preference to NADPH as co-factor compared with NADH. In addition, activity assay using H35A and K36A mutants showed significantly reduced NADPH binding and enzymatic activity confirming the importance of these two residues. Collectively, these data provide detailed structural insights into pivotal conformational changes in PaDHDPR for enzymatic catalysis and the cofactor selectivity of this important bacterial enzyme.

References:

Lee, C. W., Park, S.-H., Lee, S. G., Park, H. H., Kim, H. J., Park, H., Park, H. \& Lee, J. H. (2018). Scientific Reports.8: 7936

Keywords: dihydrodipicolinate reductase, Panisporosarcina sp. TG-14, psychrophile 